\title{
ABC1: connecting yellow tonsils, neuropathy, and very low HDL
}

Commentary

See related article

in this issue, pages R25-R31.

\author{
Helen H. Hobbs ${ }^{1}$ and Daniel J. Rader ${ }^{2}$ \\ ${ }^{1}$ Departments of Internal Medicine and Molecular Genetics, University of Texas Southwestern Medical Center at Dallas, \\ Dallas, Texas 75229, USA \\ ${ }^{2}$ Departments of Internal Medicine and Pathology and Laboratory Medicine, University of Pennsylvania, Philadelphia, \\ Pennsylvania 19104, USA
}

Address correspondence to: Helen H. Hobbs, Department of Molecular Genetics, University of Texas Southwestern Medical Center at Dallas, 5323 Harry Hines Boulevard,

Dallas, Texas 75229, USA. Phone: (214) 648-6724; Fax: (214)648-7539; E-mail: helen.hobbs@email.swmed.edu.

In 1960 , the most common surgical procedure performed on children was tonsillectomy. During one such routine operation, an astute physician recognized that the tonsils of his 5-year-old patient from Tangier Island were anything but ordinary (1). The tonsils were strikingly large, lobulated, and lemoncolored. The tonsils of the patient's sister were similarly abnormal in appearance and had a 50- to 100-fold elevated content of cholesteryl ester. The siblings also shared another remarkable trait: both had extremely low plasma levels of HDL cholesterol (HDL-C). These children were the index cases of Tangier disease, a genetic disorder characterized by an abnormal plasma lipoprotein profile virtual absence of HDL-C, reduced levels of LDL cholesterol (LDL-C) with mildly or moderately elevated triglycerides, and a variety of seemingly disparate clinical findings, such as hepatosplenomegaly and peripheral neuropathy (2). The multifaceted manifestations of this disorder have inspired (and frustrated) more clinicians and scientists than there are patients with the disease.

A breakthrough in our understanding of this enigmatic disease came recently when 3 groups identified the underlying molecular defect to be mutations in the ATP binding cassette transporter 1 (ABC1) gene (3-5). The report by Lawn et al. in this issue of the JCI extends these findings and provides a further functional characterization of the $\mathrm{ABC} 1$ transporter (6). These discoveries, which explain some of the variable clinical manifestations of Tangier disease, will provide new insights not only into the physiology and cell biology of lipid metabolism, but also into neurobiology and possibly even atherosclerosis.

$A B C 1$ and cholesterol efflux. ABC1 belongs to a large family of evolution- arily conserved transmembrane proteins that transport a wide variety of substrates, including ions, drugs, peptides, and lipids, across cell membranes (7). Mutations in other family members cause a variety of genetic disorders, such as cystic fibrosis, intrahepatic cholestasis, macular degeneration, peroxisomal dysfunction, and immunodeficiency syndromes (7). The first clue that $\mathrm{ABC} 1$ participated in lipid metabolism came with the observation by Schmitz and colleagues that $\mathrm{ABC} 1$ expression rose when the cholesterol content of macrophages was increased (8). In an independent study using expression microarrays, Lawn et al. identified the ABC1 mRNA as an upregulated transcript in cholesterol-loaded macrophages (6). Macrophages routinely ingest large amounts of cholesterol in the form of cellular membrane debris and aggregated lipoproteins, and lack the ability to downregulate cholesterol uptake in response to cholesterol loading (9). Consequently, macrophages are heavily dependent on cholesterol efflux (predominantly through transfer to HDL) to prevent accumulation of lipids. Thus, it might be anticipated that proteins that participate in cholesterol efflux, such as ABC1, would be increased in cholesterol-laden macrophages. The current finding of Lawn et al. that ABC1 is located on the cell surface suggests that this transporter may actually physically participate in the hand-off of cholesterol from cells to plasma HDL (6). This finding dovetails nicely with the previous observation that Tangier fibroblasts have a greatly reduced capacity to donate cellular cholesterol (and phospholipids) to apo A-I, the major apolipoprotein of
HDL (10). The full complement of substrates transported by $\mathrm{ABC} 1$ has not been identified, and it is possible that some of the variability in the clinical manifestations of this disease is due to mutation-specific differences in substrate specificity.

Yellow/orange tonsils. What does Tangier disease teach us about the function of $\mathrm{ABC} 1$ and the importance of facilitated cholesterol efflux in human physiology? Despite near-ubiquitous expression of ABC1 (8), the cholesterol accumulation in Tangier disease appears to be restricted primarily to macrophages and to other cells of the reticuloendothelial system. This suggests that other cell types are better able to control lipid entry and may not be as dependent on facilitated efflux. The hallmark yellow and/or orange discoloration of the tonsils, adenoids, and colonic reticuloendothelial cells in this disease may be due to the accumulation in these cells of lipophilic compounds such as vitamin $\mathrm{E}$ and retinyl esters (yellow) and carotenoids (orange). This feature of the disease now raises the possibility that $A B C 1$ is also responsible for transporting excess fat-soluble vitamins out of macrophages.

Neuropathy. In patients with Tangier disease, lipids also accumulate within Schwann cells, which synthesize and maintain the myelin sheaths of peripheral nerves. The most debilitating feature of the disease is neuropathy, which affects approximately 50\% of Tangier patients (2). Two major types of neurological syndromes are observed: (a) a syringomyelia-like disorder, with progressive loss of sensory and motor function in the upper body; and (b) a peripheral neuropathy, with remitting loss of peripheral sen- 
sory and/or motor function. It is not yet known whether different molecular defects in $\mathrm{ABC} 1$ are associated with each of these mutually exclusive neurological subtypes. The major pathological findings on peripheral nerve biopsy are neuronal loss and lipid accumulation in Schwann cells (2). The cause of nerve death in this disorder is not known, but nerve loss appears to precede the development of the lipid-laden Schwann cells (11). ABC1 may limit the accumulation of toxic substances within neurons by mediating their removal, hence providing protection from neuronal cell injury and death; precedent for such a role comes from study of another ABC family member, the multiple drug resistance 1 protein (12). Engorged Schwann cells presumably arise because of the inability of these cells to export lipids acquired from scavenged dead neurons.

Coronary artery disease. The other clinically significant sequela of defects in $\mathrm{ABC} 1$ is an increased incidence of coronary artery disease (CAD) $(13,14)$. Even obligate heterozygotes, who have reduced plasma levels of HDL-C, have an increased risk of CAD (13). The prevailing view is that subjects with Tangier disease are at higher risk for developing atherosclerosis, because of a reduction in the rate of reverse cholesterol transport, the process by which cholesterol is transported from the periphery to the liver. ABC1 participates in the first and most poorly understood step in the reverse cholesterol transport pathway - the transfer of cholesterol from cells to HDL. A defect in the function of ABC1 would be expected to result in the accumulation of cholesteryl esters in macrophages in the arterial wall, which may promote atherosclerosis. A major unanswered question is the extent to which ABC1-mediated cholesterol efflux contributes to total-body reverse cholesterol transport, a question that could be addressed using an Abc1knockout mouse. The Wisconsin HypoAlpha Mutant (WHAM) chicken may provide a natural animal model for this disorder (15).

Although the risk for early atherosclerosis is enhanced in Tangier disease, this increase is not as dramatic as might be expected $(13,14)$. As a comparison, adults with heterozygous familial hypercholesterolemia, who have markedly elevated plasma levels of LDL-C (mean $\sim 300 \mathrm{mg} / \mathrm{dL}$ ), have a 3- to 4-fold higher risk of premature CAD ( $\leq 60$ years old) than do those with Tangier disease, who have virtually no plasma HDL-C $(13,16)$. Tangier patients may have less risk than otherwise expected, because their plasma LDL-C levels are also very low ( $40 \%$ of normal, or mean plasma LDL-C $\sim 50$ $\mathrm{mg} / \mathrm{dL})(2,14)$. In epidemiological studies, individuals with plasma levels of LDL less than $\sim 80 \mathrm{mg} / \mathrm{dL}$ rarely develop CAD (17). Thus, the fact that Tangier patients develop CAD to a greater extent than do normal individuals, despite having very low LDL-C levels, suggests that impaired cellular efflux or the associated abnormalities in the other classes of lipoproteins (see below) promotes atherosclerosis.

Low HDL-C and LDL-C, and elevated triglycerides. The major cause of the markedly reduced plasma levels of HDL and apo A-I in Tangier disease appears to be an inability of HDL to acquire sufficient cholesterol from cells to maintain its metabolic integrity, resulting in markedly accelerated HDL catabolism (2). However, the reason that defective cholesterol efflux results in such low plasma levels of LDL-C remains mysterious. A reduction in HDL-mediated return of cholesterol from the periphery to the liver may alter hepatic lipid metabolism, resulting in the decreased production or lowered cholesterol content of LDL precursor lipoproteins. Alternatively, this altered metabolic pattern may promote hepatic LDL receptor activity, which would increase the rate of removal of LDL from the plasma. It is also possible that the failure to transfer cholesterol from HDL to LDL and VLDL leads to cholesterol-poor LDL, which may be metabolized more rapidly than cholesterol-rich LDL.

The metabolism of intestinally derived and hepatically derived triglyceride-rich lipoproteins (chylomicrons and VLDL, respectively) is also deranged in Tangier patients (18). These particles are markedly distorted in appearance and exceedingly variable in size, and thus may be scavenged by the reticuloendothelial system. In support of this notion, one Tangier patient experienced a doubling in his non-HDL plasma cholesterol after splenectomy (19). Initiation of a lowfat diet reduces the abundance of these abnormal particles (18) and may be associated with symptomatic improvement in the peripheral neuropathy (H.H. Hobbs, personal observation). The generalized abnormalities among all lipoprotein classes in Tangier disease illuminate the close link between the metabolism of HDL and the apo B-containing lipoproteins.

Therapeutic implications. ABC1 and the entire reverse cholesterol transport pathway present enticing targets for the development of new therapies for atherosclerosis. Although reduction of LDL-C is the crucial factor in reducing cardiovascular risk, LDL lowering fails to halt the progression of atherosclerosis in some subjects (20). Pharmacologic manipulation of the reverse cholesterol transport pathway - by increasing cellular $\mathrm{ABC} 1$ expression to augment cellular efflux of cholesterol to HDL, by preventing the catabolism of HDL without interfering with its cholesterol transport function, or by increasing the rate of hepatic HDL cholesterol uptake - may provide new strategies against a disease that is the major cause of human morbidity and mortality.

The elucidation of the molecular etiology of Tangier disease illustrates once again how the weaving together of the independent strands of basic research in cell biology and the clinical observations of a rare inherited phenotype can provide a new fabric for the understanding of a major physiological pathway.

1. Fredrickson, D.S., Altrocchi, P.H., Avioli, L.V., Goodman, D.S., and Goodman, H.C. 1961. Tangier disease: combined clinical staff conference at the National Institutes of Health. 55:1016-1031.

2. Assman, G., von Eckardstein, A., and Brewer, H.B. 1995. Familial high density lipoprotein deficiency: Tangier disease. In The metabolic and molecular bases of inherited disease. C.R. Scriver, A.L. Beaudet, and W.S. Sly, editors. McGraw-Hill. New York, NY. 2053-2072.

3. Bodzioch, M., et al. 1999. The gene encoding ATP-binding cassette transporter 1 is mutated in Tangier disease. Nat. Genet. 22:347-351.

4. Rust, S., et al. 1999. Tangier disease is caused by mutations in the gene encoding ATP-binding cassette transporter 1. Nat. Genet. 22:352-355.

5. Brooks-Wilson, A., et al. 1999. Mutations in ABC1 in Tangier disease and familial high-density lipoprotein deficiency. Nat. Genet. 22:336-345.

6. Lawn, R.M., et al. 1999. The Tangier disease gene product $\mathrm{ABC} 1$ controls the cellular apolipoprotein-mediated lipid removal pathway. J. Clin. Invest. 104:R25-R31.

7. Higgins, C.F. 1992. ABC transporters: from microorganisms to man. Annu. Rev. Cell Biol. 8:67-113.

8. Langmann, T., et al. 1999. Molecular cloning of the human ATP-binding cassette transporter 1 
(hABC1): evidence for sterol-dependent regulation in macrophages. Biochem. Biophys. Res. Commun. 257:29-33.

9. Brown, M.S., and Goldstein, J.L. 1983. Lipoprotein metabolism in the macrophage: implications for cholesterol deposition in atherosclerosis. Annu. Rev. Biochem. 52:223-261.

10. Francis, G.A., Knopp, R.H., and Oram, J.F. 1995. Defective removal of cellular cholesterol and phospholipids by apolipoprotein A-1 in Tangier disease. J. Clin. Invest. 96:78-87.

11. Antoine, J.C., et al. 1991. Pathology of roots, spinal cord and brainstem in syringomyelia-like syndrome of Tangier disease. J. Neurol. Sci. 106:179-185.

12. Ambudkar, S.V., et al. 1999. Biochemical, cellular, and pharmacological aspects of the multidrug transporter. Annu. Rev. Pharmacol. Toxicol. 39:361-398.

13. Schaefer, E.J., Zech, L.A., Schwartz, D.E., and Brewer, H.B. 1983. Coronary heart disease prevalence and other clinical features in familial high-density lipoprotein deficiency (Tangier disease). Ann. Intern. Med. 93:261-266.

14. Serfaty-Lacrosniere, C., et al. 1994. Homozygous Tangier disease and cardiovascular disease. Atherosclerosis. 107:85-98.

15. Poernama, F., Schreyer, S.A., Bitgood, J.J., Cook, M.E., and Attie, A.D. 1990. Spontaneous high density lipoprotein deficiency syndrome associated with a $Z$-linked mutation in chickens. J. Lipid Res. 31:955-963.
16. Stone, N.J., Levy, R.I., Fredrickson, D.S., and Verter, J. 1974. Coronary artery disease in 116 kindreds with familial type II hyperlipoproteinemia. Circula tion. 49:476-488.

17. Gordon, T., Kannel, W.B., Castelli, W.P., and Dawber, T.R. 1981. Lipoproteins, cardiovascular disease, and death. The Framingham study. Arch. Intern. Med. 141:1128-1131.

18. Herbert, P.N., Forte, T., Heinen, R.J., and Fredrickson, D.S. 1978. Tangier disease: one explanation of lipid storage. N. Engl. J. Med. 299:519-521.

19. Schaefer, E.J., et al. 1983. Massive omental reticuloendothelial cell lipid uptake in tangier disease after splenectomy. Am. J. Med. 75:521-526.

20. Superko, H.R. 1996. Beyond LDL cholesterol reduction. Circulation. 94:2351-2354. 